BRES 18198

\title{
Functional regeneration of glossopharyngeal nerve through micromachined sieve electrode arrays
}

\author{
Robert M. Bradley ${ }^{a, b}$, Richard H. Smoke a ${ }^{\text {, Tayfun Akin }}{ }^{\mathrm{c}}$ and Khalil Najafi ${ }^{\mathrm{c}}$ \\ a Department of Biologic and Materials Sciences, School of Dentistry, Unilersity of Michigan, Ann Arbor, MI (USA), ' Department of Physiology, \\ School of Medicine, Unitersity of Michigan, Ann Arbor, MI (USA) and ' Center for Integrated Sensors and Circuits, Department of Electrical \\ Engineering and Computer Science, Unitersity of Michigan, Ann Arbor, MI (USA)
}

(Accepted 26 May 1992)

Key words: Glossopharyngeal nerve; Sieve electrode array; Electrophysiology; Taste; Regeneration; Neural prosthesis; Chronic recording

\begin{abstract}
To assess the potential of micromachined silicon sieve electrodes for long term recordings from single afferent sensory fibers, we implanted them between the cut ends of rat glossopharyngeal nerves which innervate taste and somatosensory receptors on the posterior tongue. After the implants had been in place for an average of 101 days nerve regeneration was measured using histological and electrophysiological methods. Axons of the glossopharyngeal nerve regenerated through holes in the sieves and supported the functional regeneration of taste, thermal and mechanoreceptors.
\end{abstract}

\section{INTRODUCTION}

Neural information is transmitted from sensory receptors over many fibers, and thus sensory coding of the peripheral stimulus is contained in both the temporal firing pattern of individual fibers and the parallel activity of many fibers in an across-fiber pattern ${ }^{4}$. The usual approach to studies of across-fiber patterns is to record from many, separate single fibers, often in a number of different animals, and then to examine across-fiber patterns in this array of single fibcr recordings. The assumption is made that this compilation of single fiber recordings represents the across-fiber pattern that would have been obtained if all these fibers had been recorded simultaneously.

To permit parallel simultaneous recording from a number of fibers, several investigators have suggested the use of a sieve electrode. The basic idea is to manufacture a thin diaphragm with many small holes that can be positioned between the cut ends of a peripheral nerve. The nerve is then left to regenerate through the small holes to reinnervate its target organs". If electrode sites are associated with some or all of the holes, then it becomes possible to record from single nerve fibers that have regenerated through the holes.

A number of these devices have been fabricated using different technical approaches including mechanical and laser drilling and semiconductor fabrication, incorporating wet and dry etching of silicon substrates $3, x, 11,12$. While early designs were passive, the most recent ones have the potential to incorporate active electronics on the device. Some successful recordings have been obtained ${ }^{8.12}$, but other designs were not successful or were not tested in vivo" ${ }^{11}$. Other problems with earlier devices included the relatively large diameters of fabricated holes $(>8 \mu \mathrm{m})$, limitations on minimal achievable substrate thickness ( $>100$ $\mu \mathrm{m})$. low density of holes, problems with connecting the electrode sites to the recording equipment and the complexity of tie fabrication process. We now report on the in vivo testing of a micromachined silicon sieve electrode that overcomes many of the limitations of earlier designs. in the experiments reported here we evaluated whether nerve fibers would regenerate through the electrode and form functional connections with peripheral sensory receptors. 


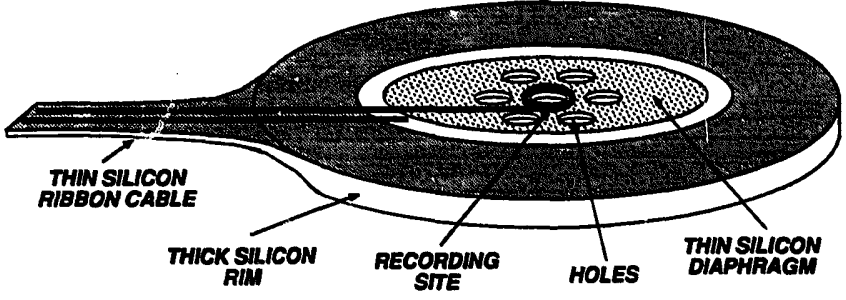

Fig. 1. Diagram of the essential features of the sieve electrodes.

\section{MATERIALS AND METHODS}

To assess the potential of this new sieve electrode design, we fabricated a series of the sieve electrodes with different hole configurations, and implanted them between the cut ends of the glossopharyngeal nerve which innervates taste and somatosensory receptors on the posterior tongue. After a suitable survival time we examined whether functional regeneration of the nerve and sensory receptors it supplies had occurred. We chose to use the rat glossopharyngeal nerve because numerous investigators have demonstrated the regenerative capacity of this nerve (see review by Oakley ${ }^{14}$ ) and because we will eventually use the implant to answer a number of fundamental questions regarding the cell biology of taste receptors. In the present experiments we did not attempt to record from electrode sites on the implanted devices.

Details of the electrode structure and fabrication process already have been described'. Briefly the electrode consists of a $15 \mu \mathrm{m}$ thick silicon support rim and a $4 \mu \mathrm{m}$ thick silicon diaphragm which contains a number of holes of different sizes (Fig. 1). Both the support rim and diaphragm are formed by thermal diffusion of boron into a silicon wafer, and then dissolving the undoped regions of the silicon wafer in a silicon etchant leaving only the boron-diffused regions. This allows precise control of the shape and thickness of both the diaphragm and support rim. Holes in the diaphragm are manufactured by selectively masking against boron diffusion in these areas. Thus, a variety of different sizes and densities of holes can be formed by simply modifying the diaphragm diffusion mask pattern. The boron-doped silicon substrate supports an array of conductors made of phosphorous-doped polycrystalline silicon which are insulated above and below by deposited silicon dioxide and silicon nitride dielectrics. These conductors interconnect bonding pads, located some distance from the nerve regeneration electrode area, and the recording sites that are located around some of the holes in the central diaphragm. Recording sites are formed by selectively removing the top dielectric layers down to the polysilicon conductors and depositing iridium in these exposed areas (Fig. 2A). It is also possible to integrate a multi-lead ribbon cable with the electrode to interconnect the recording sites and the bonding pads at the opposite end of the electrode (Fig. 2B,C). All of the materials used in fabricating the sieve electrode, including the boron-doped silicon substrate, silicon dioxide, silicon nitride, and iridium electrode sites have been shown to be biocompatible and can withstand long-term exposure to corrosive body fluids. In addition, the silicon substrate used in fabricating these electrodes is very robust and flexible.

In an attempt to match hole configurations to the fiber diameters of the glossopharyngeal nerve we took advantage of the versatility of the fabrication process and produced several different combinations of hole sizes. Designs include sieve electrodes with large numbers of small diameter $(2 \mu \mathrm{m})$ holes and center-spacing as small as $10 \mu \mathrm{m}$, as well as various combinations of large and small diameter holes (Table 1).

Once fabricated, short lengths $(1.0 \mathrm{~mm})$ of $0.3 \mathrm{~mm}$, inside dianieter, silicone tubing were cemented onto either side of the diaphragm with silicone adhesive. These acted as guide tubes to facilitate positioning and fixation of the cut ends of the glossopharyngeal nerve. Large holes in the sieve electrode support rim were provided to aid fixation of the guide tubes (Fig. 2B).
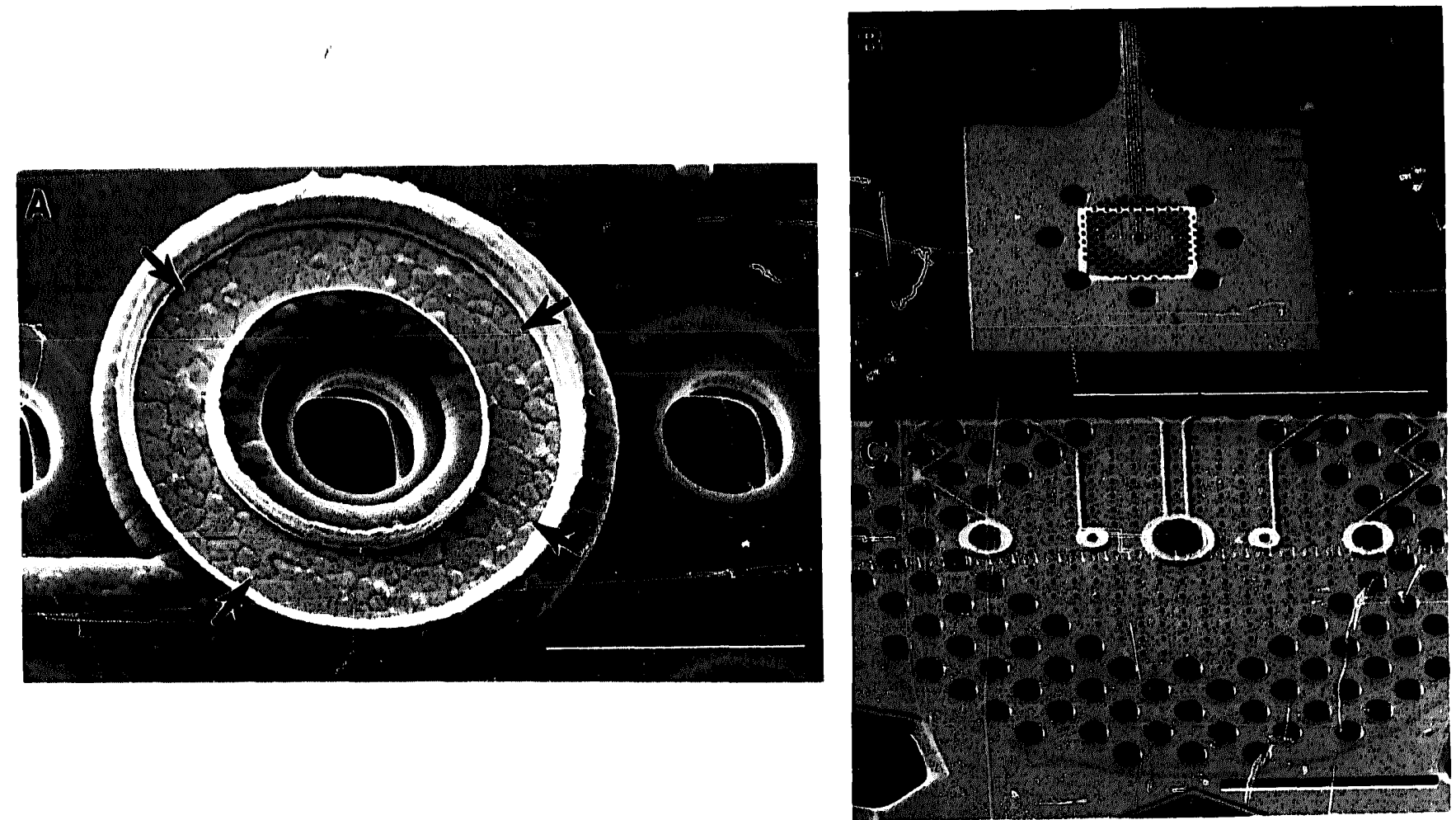

Fig. 2. A: scanning electron micrograph of a $5 \mu \mathrm{m}$ diameter hole surrounded by an iridium recording site (arrows). Bar $=10 \mu \mathrm{m}$. B: low-power scanning electron micrograph of a sieve electrode showing the central diaphragm area with large and small holes surrounded by the thicker rim with large holes used to position and cement the nerve guide tubes. The integrated ribbon cable can be sean at the top of the sieve electrode. Bar $=1 \mathrm{~mm}$. C: higher magnification scanning electron micrograph of the area outlined in (B) showing the two sizes of holes, elertrode sites and connecting conductors. Bar $=100 \mu \mathrm{m}$. 
TABLE I

Number and diameters of holes in the different siecc electrode designs

\begin{tabular}{lcccc}
\hline Design & $\begin{array}{l}\text { No. } 2 \mu m \\
\text { holes }\end{array}$ & $\begin{array}{l}\text { No. } 5 \mu \mathrm{m} \\
\text { holes }\end{array}$ & $\begin{array}{l}\text { No. } 10 \mu \mathrm{m} \\
\text { holes }\end{array}$ & $\begin{array}{l}\text { No. } 15 \mu \mathrm{m} \\
\text { holes }\end{array}$ \\
\hline 1 & 151 & - & - & - \\
2 & $77 \%$ & - & - & - \\
3 & - & 497 & - & - \\
4 & 540 & 1 & - & 52 \\
5 & 197 & - & - & 133 \\
6 & - & 174 & - & 133 \\
7 & 286 & - & - & - \\
8 & - & 214 & - & - \\
9 & - & - & 131 & - \\
10 & 5 & 130 & 170 & - \\
\hline
\end{tabular}

Rats were anesthetized with an intramuscular injection of a $1: 1$ mixture of Rompun ( $2 \mathrm{mg} / \mathrm{kg}$ b.wt.) and ketamine $(10 \mathrm{mg} / \mathrm{kg})$ and laid supine on a heating pad. A midline incision was made in the skin overlying the trachea, and the glossopharyngeal nerve exposed by removal of the hyoid process. The nerve was cut close to the base of the tongue and the cut ends positioned in the silicone guide tubes on either side of the silicon sieve. The nerves were secured in place with 10-0 monofilament suture passed through the perineurium and the wall of the guide tubes. The wounds were then closed and the rat returned to its home cage.

After a survival time of at least 90 days the rats were reoperated 10 assess the extent of regeneration by recording from the glossopharyngeal nerve central to the implant. Rats were anesthetized with an intraperitoneal injection of sodium pentobarbital $(50 \mathrm{mg} / \mathrm{kg} \mathrm{b.wt.)}$ and given supplemental doses as necessary. The rats were tracheotomized and placed on a heating pad. The glossopharyngeal nerve central to the implant was carefully dissected and cut close to its exit from the skull. Both hypoglossal nerves were cut to eliminate reflex tongue movements. The skin and muscle of the side of the mouth was dissected to reveul the lateral border of the tongue contuining the foliate papitlac. With the rat positioned on its side the nerve was lifted onto a pair of platinum-iridium recording electrodes connested to an $\mathrm{AC}$ preamplifier with a reference electrode in nearby tissue. Neural activity from the whole nerve wus analyzed by passing through an AC.DC converter (time constant 0.5 s) connected to a pen recorder.

Stimuli were $1.0 \mathrm{M} \mathrm{NaCl}, \mathrm{NH}_{4} \mathrm{Cl}, \mathrm{KCl}, \mathrm{Na}$-Saccharin, $0.01 \mathrm{M}$ Qu-HCl and $0.1 \mathrm{M}$ eitric acid dissolved in distilled water and maintained at $37^{\circ} \mathrm{C}$, as well as ice cold water (thermal stimulus) and a glass rod (mechanical stimulus). The chemical stimuli were flowed over the posterior tongue using a syringe for abont $1 \mathrm{~min}$. Stimuli were rinsed with $37^{\circ} \mathrm{C}$ distilled water At least 1 min elapsed before another stimulus was applied.

After the electrophysiological recording the glossopharyngeal nerve distal to the implant was cut and a block of tissue containing the implant removed. Under a dissecting microscope the implant containing the glossopharyngeal nerve was carefully isolated by removing the surrounding scar and connective tissue and then pholographed. After fixation in $2 \%$ paraformaluehyde in $0.1 \mathrm{M}$ phosphate buffer the entire implant was embedded in epoxy resin and 1 $\mu \mathrm{m}$ thick plastic sections cut of the distal glossopharyngeal nerve stump. These were stained with $1 \%$ Toluidine blue and examined for the presence of regenerated nerve fibers. The posterior tongue was also removed, fixed in 10\% neutral-buffered formalin, embedded in paraffin, sectioned at $10 \mu \mathrm{m}$, stained with heinatoxylin and eosin and then examined for the presence of taste buds in the foliate papillae.

We examined the foliate papillae because the posterior clefts of this papilla are unilaterally innervated by the glossopharyngeal nerve ${ }^{13}$. By comparing the number of taste buds on the operated and unoperated sides of the tongue, estimates of taste bud regeneration were made. Foliate papilla taste buds were quantified by counting every profile of a taste bud in each scrial section of the papitia and then dividing this total by the average number of sections occupied by a taste bud determined from careful examination of 10 individual taste buds².

\section{RESULTS}

Sieve electrodes were implanted in 28 rats for times ranging from 91 to 118 days $(\vec{x}=101 \pm 6.4$, S.D. $)$. Based on gross examination of the implants and histological examination of the distal nerve cross-sections, regeneration through the sieve occurred in 21 of the implants. In the remaining 7 animals, either the implant was empty or very few fibers were present in the distal nerve section. When regeneration was successful, the glossopharyngeal nerve was clearly visible in the guide tubes (Fig. 3, arrowheads) and numerous myelinated fibers were present in the cross-section of the nerve (Fig. 4). In unsuccessful regenerations the nerve was often observed to have bypassed the implant, presumably because it pulled out of the guide tubes. No systematic difference was observed in the extent of regeneration with the use of different hole configurations of the sieve design.

Electrophysiological recording from the central end of the regenerated glossopharyngeal nerve was difficult because a considerable amount of scar tissue surrounded the implant. However, recordings were made from 19 of the 21 animals with confirmed regeneration of the nerve through the implant. Responses to mechanical, thermal and chemical stimulation of the posterior tongue were obtained in all the recordings. The signal-to-noise ratio was inferior in many of the recordings, especially the responses to chemical stimuli. However, good signal-to-noise ratio responses to chemical stimulation were obtained in 5 animals. Two examples of these recordings illustrating responses to taste stimuli, as well as thermal and mechanical stimuli, are shown in Fig. 5A,B. These responses are similar to whole nerve recordings from an intact glossopharyngeal nerve (Fig. 5C).

Counts of foliate taste buds were made on 14 tongues. There was ivide variability in the number of taste buds on both the control and operated sides of

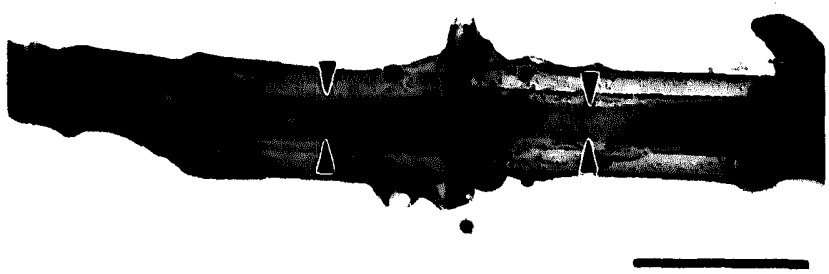

Fig. 3. Low-power photomicrograph of an implant after removal. The regenerated glossopharyngeal nerve (arrowheads) can be seen in place in the silicone guide iubes. Bar $=1 \mathrm{~mm}$. 
the tongues. Regenerated taste buds ranged in number from 17 to 207 with a mean of $94 \pm 70$ (S.D.) while number of taste buds on the control side varied between 91 and $232(\bar{x}=179 \pm 43$, S.D.). The small number of taste buds on the side of the tongue ipsilateral to the implant observed in some animals may not in fact be regenerated taste buds but represent taste buds innervated by the chorda tympani nerve, since Oakley has shown that 17 taste buds remain in the rat foliate papilla when the glossopharyngeal nerve is cut ${ }^{13}$. In 6 animals the number of taste buds on the regenerated side was equivalent to the unoperated control side, indicating that control numbers of taste buds could regenerate (Fig. 6).

\section{DISCUSSION}

It is apparent from these experiments that axons of the glossopharyngeal nerve will regenerate through 2 $\mu \mathrm{m}$ diameter holes and support the regeneration of taste buds. The regenerated taste buds are functional and respond to chemical stimulation of the tongue. Thermal and mechanoreceptors also become reinnervated and functional.

Although the sieves contain a large number of holes (e.g. 777, $2 \mu \mathrm{m}$ diameter holes in design Na. 2) there are many more fibers in the glossopharyngeal nerve. Jang and Davis ${ }^{7}$ have measured and counted axons in the hamster glossopharyngeal nerve which has an average of $1,604 \pm 47$ (S.E.) axons ranging in diameter from
0.2 to $2.4 \mu \mathrm{m}$. Most of these axons (79\%) are unmyelinated with a mean diameter of $0.5 \mu \mathrm{m}$. Jang and Davis also report observations of I.J. Miller Jr. on the rat glossopharyngeal nerve which contains about 1,900 axons, $53 \%$ of which are myelinated. It is reasonable to assume from these anatomical studies that there are approximatcly 2-3 axons for every hole in the sieves. At present we do not know how many axons pass through each hole, but because of the large numbers of holes in these sieves, some holes may have several fibers, while others may only contain one. Moreover, since no differences were observed between the extent of regeneration with the different sieve designs, we will use the sieve with the highest density of holes for chronic recording to maximize the potential of single or few fiber recordings.

Based on taste bud counts in the foliate papillae there is wide variability in the extent of taste bud regeneration. Part of this variability is no doubt related to surgical expertise, as the number of regenerated taste buds was much lower with the earlier implants. For example, there was a mean of 59 taste buds in 5 foliate papillae of the initiai implants and a mean of 144 taste buds in the last 5 implanted animals. Furthermore, no attempt was made to improve the chances of nerve regeneration by using substances known to promote nerve regeneration in silicone chambers such as nerve growth factor, laminin and other putative neurotrophic factors ${ }^{5,6}$. Perhaps in future experiments, application of substances such as Imininin might improve

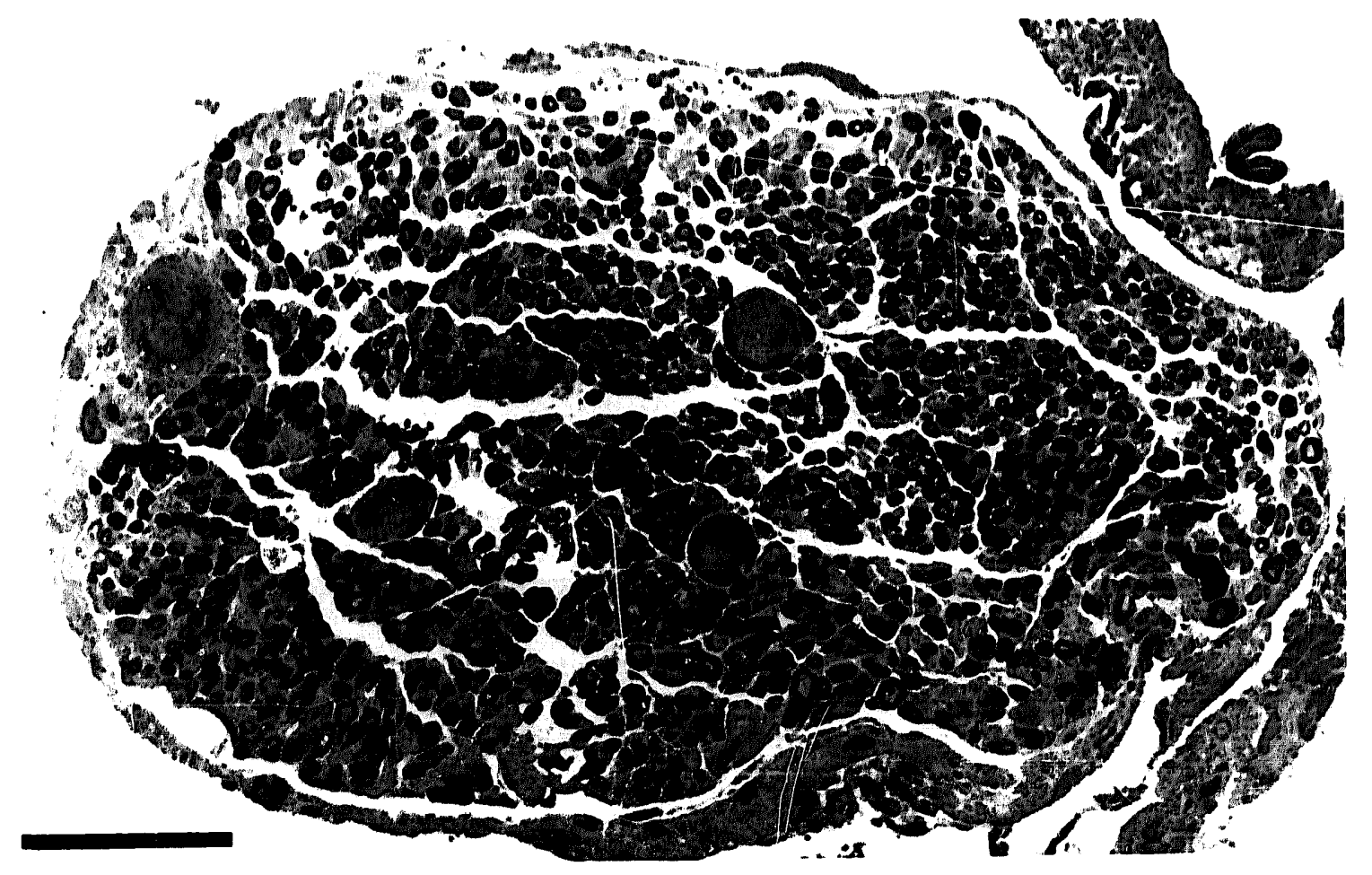

Fig. 4. Photomicrograph of distal nerve cross-section showing large numbers of myelinated nerve fibers. Bar $=100 \mu \mathrm{m}$. 
the extent and reduce the time required for regeneration. However, in many investigations of nerve regeneration, the object is to get the nerve to regenerate across a large gap, to serve as a model for surgical trauma repair. The separation provided by the sieve electrode is very small $(4 \mu \mathrm{m})$ so that the nerve has to regenerate across a very small gap.

It is evident that these newly developed sieve electrodes with integral ribbon cable connecting leads have the potential for chronic recording from single nerve fibers. Although the number of electrodes associated with the holes in the sieves is currently limited to 5 , it will be relatively simple to add active electronics to the device to allow switching between electrodes to effectively increase the number of electrodes on each sieve.
This will be important not only to examine across-fiber patterns but also to select the electrodes with the most favorable signal-to-noise ratio. In addition, because the glossopharyngeal nerve carries afferent information from taste buds, thermal and mechanoreceptors it will be possible to select and study particular sensory modalities within the nerve. It will also be possible to eliminate non-relevant neural information because the glossopharyngeal contains many parasympathetic efferent fibers supplying secretory elements in the tongue.

Sieve electrodes can also be used as the neural interface to a prosthetic device. Indeed many of past as well as the current laboratories developing these devices are doing so because of their potential as a neural prosthesis ${ }^{8,10,11}$. The present experiments, therefore,

A

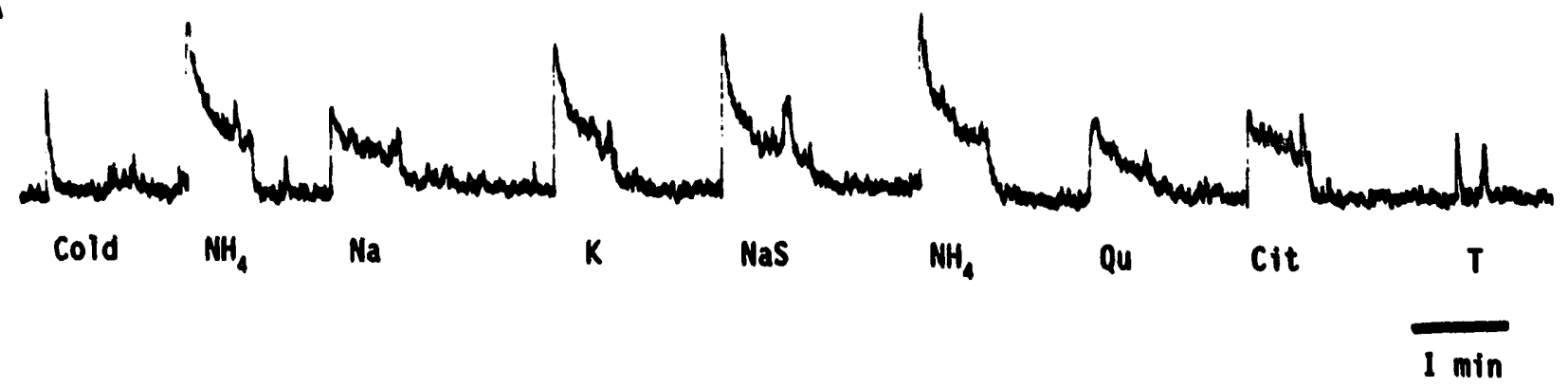

B

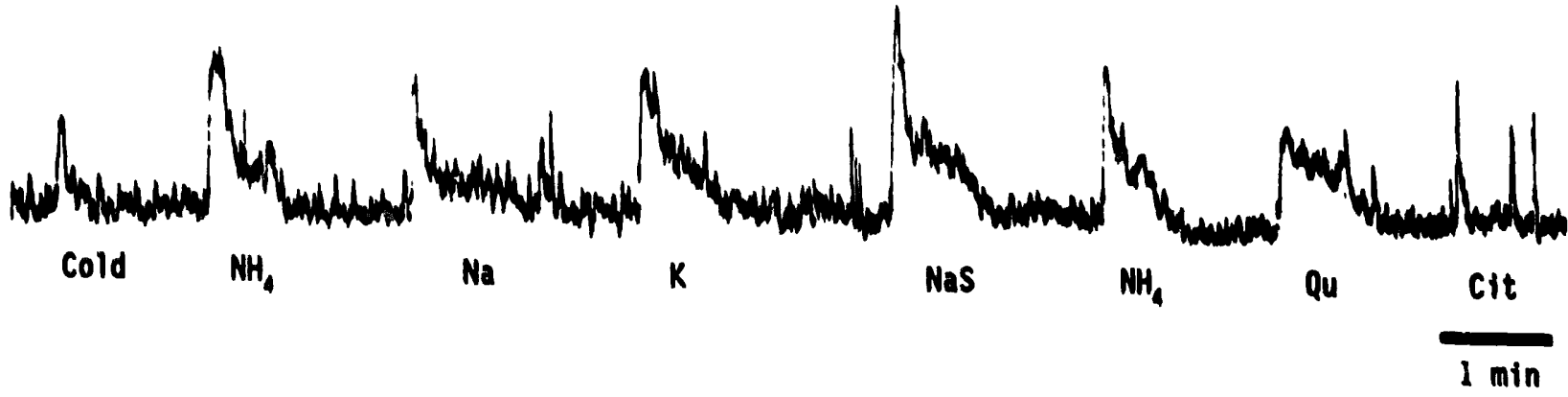

C

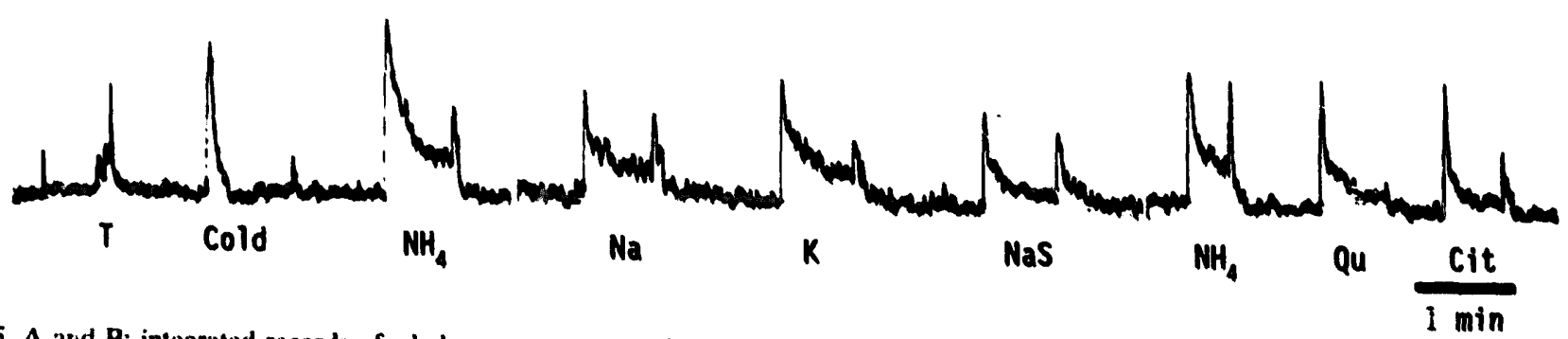

Fig. 5. A and B: integrated records of whole nerve responses from glossopharyngeal nerves that had regenerated through sieve electrodes. C: integrated records of whole nerve responses from an unoperated glossopharyngeal nerve. Cold, ice cold water thermal stimulus; $\mathrm{T}$, glass rod mechanical stimulus; $\mathrm{NH}_{4}, 1.0 \mathrm{M} \mathrm{NH}+\mathrm{Cl} ; \mathrm{Na}, 1.0 \mathrm{M} \mathrm{NaCl} ; \mathrm{K}, 1.0 \mathrm{M} \mathrm{KCl} ; \mathrm{NaS}, 1.0 \mathrm{M} \mathrm{Na-Saccharin;} \mathrm{Qu,} \mathrm{0.01} \mathrm{M} \mathrm{Quinine-HCl;} \mathrm{Cit,} \mathrm{0.1} \mathrm{M} \mathrm{sitric}$ acid. 

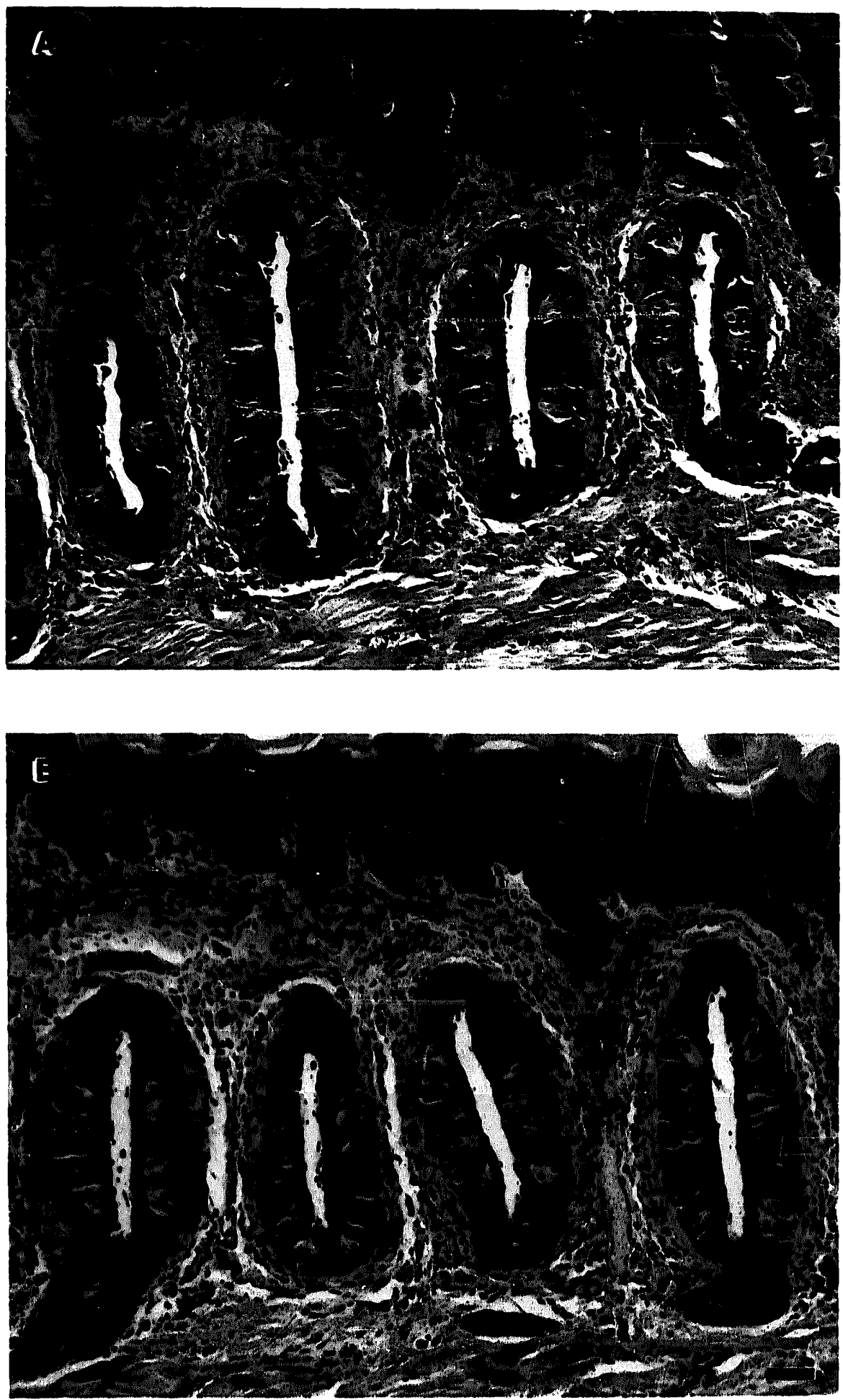

Fig. 6. Photomicrograph of saggital sections through the foliate papillae of control (A) and regenerated (B) sides of a tongue. Bar $=100 \mu \mathrm{m}$.

will have significant use in the study of sensory coding as well as providing valuable information on the design of prosthetic devices.

Acknowledgements. This work was supported by National Institutes of Health Grant DC00059 to RMB.

\section{REFERENCES}

1 Akin, T. and Najafi, K., A micromachined silicon sieve electrode for nerve regeneration applications. In Digest of Technical Papers, International Conference on Solid-State Sensors and Actuators, IEEE, 1991, pp. 128-131.

2 Bradley, R.M., Cheal, M.L. and Kim, Y.H., Quantitative analysis 
of developing epiglottal taste buds in sheep, J. Anat., 130 (1980) 25-32.

3 Brindley, G.S., Electrode-arrays for making long-lasting electrical connexion to spinal rovts, J. Physiol., 222 (1972) 135-136.

4 Erickson, R.P., Parallel "population" neural cocing and feature extraction. In F.O. Schmitt and F.G. Worden (Eds.), The Neurosciences, Third Study Program, MIT Press, Cambridge, 1974, pp. 155-169.

5 Harmas, K., Kätnick, J., Lim. R., Zaheer, A. and De la Torre, J.C.. Glia maturation factor $\beta$ stimulates axon regeneration in transected rat sciatic nerve, Brain Res., 564 (1991) 332-335.

6 Hollowell, J.P., Villadiego, A. and Rich. K.M., Sciatic nerve regeneration across gaps within silicone chambers: long-term effects of NGF and consideration of axonal branching, Exp. Neurol., 110 (1990) 45-51.

7 Jang. T. and Davis, B.J.. The chorda tympani and glossopharyngeal ne rves in the adult hamster, Chem. Senses, 12 (1987) 381-395.

8 Kovacs, G.T.A., Storment, C.W., Belczynski, C.R., Nguyen, K.D. and Rosen, J.M.. Thin-film microelectrode array for peripheral nerve recording and stimulation, Neurosci. Abstr., 17 (1991) p. 567.
9 Llinás, R., Nicholson, C. and Johnson, K., Implantable monolitiic wafer recording electrodes for neurophysiology. In M.I. Phillips (Ed.), Brain Unit Acticity During Behatior, Thomas, Springfield, Illinois, 1973, pp. 105-111.

10 Loeb, G.E., Neural prosthetic interfaces with the nervous system, Trends Neurosci., 12 (1989) 195-201.

11 Loeb, G.E., Marks, W.B. and Beatty, P.G., Analysis and microelectronic design of tubular electrode arrays intended for chronic, multiple single-unit recordings from captured nerve fibers, Med. Biol. Eng. Computing, 15 (1977) 195-201.

12 Mannard, A., Stein, R.B. and Charles, D., Regeneration electrodes units: implants for recording from single peripheral nerve fibers in freely moving animals, Science, 183 (1974) 547-549.

13 Oakley, B., On the specification of taste neurons in the rat tongue, Brain Res., 75 (1974) 85-96.

14 Oakley, B., Trophic competence in mammalian gustation. In D.W. Pfaff (Ed.), Taste, Olfaction, and the Central Nerious System, Rockefeller University Press, New York, 1985, pp. 92-103. 